\title{
ARTÍCULO RESEÑA
}

\section{Palestina/Israel: reproducción de pautas históricas}

\section{Palestine/Israel: A Reproduction of Historical Patterns}

\author{
JOSÉ ABU-TARBUSH QUEVEDO \\ Universidad de La Laguna, España
}

Resumen: Este trabajo es una reseña crítica del libro de Jorge Ramos Tolosa: Los años clave de Palestina-Israel. Pablo de Azcárate y la ONU (1947-1952). Siguiendo el hilo conductor de las misiones de este diplomático español, el autor analiza un periodo crucial de la historia centrado en la transformación geopolítica y demográfica de Palestina y la implantación del Estado israelí, proceso impulsado inicialmente por Gran Bretaña y secundado en la posguerra mundial por las superpotencias, Estados Unidos y la Unión Soviética, sin olvidar la responsabilidad de las Naciones Unidas por su descuido

Recepción: 27 de noviembre de 2020. / Aceptación: 11 de mayo de 2021. 
en la preservación de la paz y por la parcialidad de algunos de sus órganos y personalidades. En una lectura comparativa con la literatura especializada, se advierten ciertas pautas de comportamiento reproducidas hasta hoy: asimetría de poder entre las partes, apoyo y parcialidad de la potencia predominante en el sistema internacional, e instrumentalización de las instituciones internacionales.

Palabras clave: Palestina/Israel; Gran Bretaña; Naciones Unidas; mediación internacional.

Abstract: This work is a critical book review of Jorge Ramos Tolosa's Los años clave de Palestina-Israel. Pablo de Azcárate y la ONU (1947-1952). The missions of this Spanish diplomat provide the basis for this analysis of a crucial historical period that focuses on the geopolitical and demographic transformation of Palestine and the establishment of the Israeli State-a process initiated by Great Britain and continued by both superpowers, the United States and the Soviet Union, after the Second World War. The United Nations was also partly responsible by neglecting its duty to preserve the peace and through the bias of some parts of its organization and its prominent figures. A comparative study of specialized literature reveals patterns of behavior still in evidence today: an asymmetry of power between the parties, support, and bias of the hegemonic international power and the instrumentalization of international institutions.

Keywords: Palestine/Israel; Great Britain; United Nations; international mediation.

La historia del conflicto que enfrenta al Estado israelí con la población autóctona de Palestina tiene numerosas aristas, algunas tan peculiares como las que narra el historiador Jorge Ramos Tolosa en Los años clave de Palestina-Israel. Pablo de Azcárate y la ONU (1947-1952) (Marcial Pons Historia, 2019), referidas a la misión que protagonizó el diplomático español Pablo de Azcárate en dicho conflicto, con una biografía profesional y política no menos curiosa. Después de 15 años en la Sociedad de Naciones, donde llegó a ser secretario adjunto, fue emba- 
jador de España en Londres durante la Segunda República y la guerra civil española (1936-1939), sin haber conseguido que el Reino Unido abandonara su política de no intervención pese a la injerencia de Alemania e Italia. Sin esperanza de restitución de la República tras la Segunda Guerra Mundial, Azcárate fue invitado a retomar su carrera internacional con su ingreso en la recién constituida Organización de las Naciones Unidas (ONU).

En esta corta e intensa andadura (1947-1952), el diplomático español asumió sucesivas responsabilidades en la Comisión de Palestina, en la Comisión Consular de la Tregua, como comisario municipal interino de Jerusalén y como representante del mediador, y, finalmente, en la Comisión de Conciliación de Palestina. Pese a su rigor y su profesionalidad, sostiene el profesor Ramos Tolosa, Azcárate pertenecía a una escuela diplomática anterior, de corte liberal, distinta a la que emergió tras la Segunda Guerra Mundial, con importantes transformaciones en el manejo de los tiempos y los medios de comunicación. Su fuerte era "una diplomacia más personal y más cercana a los centros de poder" que una sobre el terreno, en zonas y situaciones de conflicto. Se sentía más cómodo en los ámbitos restringidos de la élite diplomática, de negociaciones discretas o secretas, que en la naciente diplomacia pública, más expuesta a la presión del escrutinio mediático y de la opinión pública (Ramos 2019, 67-68). Su concepción del trabajo diplomático era más formal y clásica, y pareció resentirse ante cierta dejación, escasa dotación de medios y falta de neutralidad de sus superiores en las comisiones en las que estuvo adscrito. Así lo manifestó en algunos de los testimonios que dejó en formato de libros (Azcárate, 1968), diario e informes, y que han sido consultados por Ramos Tolosa, junto con otros archivos y fuentes de primera y segunda mano.

Como actor individual, el peso de Azcárate resulta anecdótico frente al de la ONU, las potencias mundiales y otros actores regionales, pero su testimonio ofrece, en palabras de Ramos Tolosa (2019, 426), "unas perspectivas extraordina- 
rias para comprender el papel de la ONU en Palestina y la propia cuestión israelo-palestina". De hecho, el hilo conductor de esta obra son las sucesivas misiones que desempeñó en las mencionadas comisiones durante poco más de un lustro, en el que conoció diversos acontecimientos trascendentales: adopción de la Resolución 181 (II) de partición del territorio palestino (29 de noviembre de 1947), gradual limpieza étnica de Palestina (1947-1949), proclamación del Estado de Israel (14 de mayo de 1948), primer conflicto interestatal árabe-israelí (1948), adopción de la Resolución 194 (III) sobre el derecho al retorno de los refugiados palestinos (1949), y negociaciones y acuerdos de armisticio entre los Estados árabes e Israel (1948-1952).

El fracaso fue el denominador común de las misiones desempeñadas por Azcárate, no por falta de pericia diplomática, sino por una combinación de actores y factores que marcaron el futuro de la región. Ramos Tolosa destaca la falta de experiencia tanto de la ONU en la mediación y la resolución de conflictos a la hora de abordar la cuestión de Palestina (era la primera controversia que asumía en su agenda) como la de los otros actores. En primer lugar, la impericia de Gran Bretaña, considerada la principal potencia mundial y colonial de la época, con una evidente responsabilidad política e histórica al avalar la empresa colonial sionista en Palestina mediante la Declaración Balfour, en 1917, en la que se comprometía a realizar "sus mejores esfuerzos" para "el establecimiento en Palestina de un hogar nacional para el pueblo judío”. Conviene recordar que en ese entonces Londres no poseía ningún vínculo jurídico ni político con Palestina, salvo el que derivaba de su fuerza como potencia mundial que ocupó el territorio palestino en el transcurso de la Gran Guerra.

Ambos actores convergieron en sus respectivos intereses. Gran Bretaña deseaba ampliar y reforzar su Imperio colonial ante la creciente competición de las grandes potencias, derivada de la reconfiguración de la naturaleza y los intereses imperialistas (Regan 2017), y el movimiento sionista buscaba 
el amparo de una potencia mundial con influencia efectiva en la región del Cercano Oriente -en concreto en Palestinapara implementar su proyecto colonial. En suma, el gobierno británico otorgaba lo que entonces se conocía como "carta colonial”, que concedía el derecho de apropiación y explotación de un territorio a una empresa colonial.

No menos controvertido fue que, al asumir oficialmente el Mandato de la Sociedad de Naciones sobre Palestina en 1922, Londres incorporó la Declaración Balfour al objetivo de su Mandato (de tipo A), lo cual contradecía el propósito de conducir a la independencia a los países que, por el grado de desarrollo que habían alcanzado, la potencia mandataria tutorizaba. Más allá del subterfugio neocolonial que suponía la propia figura del Mandato, Gran Bretaña hizo justo lo contrario de lo que tenía que haber sido su cometido. En lugar de conducir a la independencia a la sociedad y el territorio palestino, impuso numerosos obstáculos para capacitar su desarrollo político, económico y social; impidió la construcción de sus instituciones nacionales y protoestatales; negó la representación democrática de toda la población (debido al equilibrio demográfico mayoritariamente árabe ante las pequeñas comunidades judías establecidas en el país e incluso las más numerosas y recientes oleadas inmigratorias de judíos europeos), y reprimió con dureza las manifestaciones de protestas y la rebelión anticolonial palestina (1936-1939), con el consecuente desmantelamiento de su movimiento nacional. Al mismo tiempo que negaba los medios y el derecho a la autodeterminación palestina, Londres auspició significativamente la transformación demográfica y geopolítica de Palestina mediante el apadrinamiento de la creciente colonización sionista, la política de continua y masiva inmigración judía, el reconocimiento político del movimiento sionista y, en definitiva, su imprescindible colaboración para la construcción del embrión institucional y paraestatal sionista.

Con esta trayectoria no sorprende que uno de los principales obstáculos con los que tropezó Azcárate al frente de la 
Comisión de Palestina -como afirma Ramos Tolosa (2019, 150) - fuera la reticencia de la potencia mandataria a colaborar con la ONU. El objetivo de su primera misión era "asegurar el traspaso de poderes del Mandato a la Comisión y, a su vez, de ésta a los consejos provisionales del gobierno". Sin embargo, la realidad que se impuso sobre el terreno fue otra muy distinta. Ni la potencia mandataria, ni las grandes potencias ni, por extensión, la ONU, adoptaron las medidas necesarias para prevenir el conflicto que se avecinaba ante la inminente partición del territorio palestino. Como señala el autor de Los años clave de Palestina-Israel, Gran Bretaña estuvo más centrada en "atenuar el enfrentamiento sionista-palestino y minimizar sus propias bajas", en la creencia de que "la llegada de una comisión que tenía como finalidad aplicar la partición agudizase la violencia entre las dos comunidades" (149).

Convendría matizar esta posición, teóricamente bien intencionada, y contrastarla con su política mandataria para advertir que se trataba de una deliberada pasividad. Sólo cabe remitirse al precedente más inmediato del periodo de entreguerras, que registró una lucha asimétrica entre el incipiente movimiento nacional palestino (MNP), por un lado, y el movimiento sionista y Gran Bretaña, por el otro. La reiterada frustración del MNP ante la parcialidad y la connivencia británica terminó en una temprana rebelión anticolonial (1936-1939) que pagó un precio muy alto por la fuerte represión británica y la consiguiente devastación y descabezamiento del MNP, en un momento crucial de su historia política marcado por la decisión de partir su territorio. En esta tesitura, el MNP vio malogrados sus objetivos de acceder a la independencia y a un Estado soberano. Como indica el historiador Rashid Khalidi $(2007,125)$, tampoco consiguió articular una representación nacional. La sociedad palestina dejó de ser un actor clave, fue deliberadamente ignorada al no ser consultada sobre su futuro y perdió su condición de "agencia" en favor del naciente Estado israelí, los Estados árabes vecinos y otros actores internacionales. En suma, pese a las diferencias y las contradicciones entre el liderazgo sionista 
y el Mandato británico en Palestina, en particular a raíz de la adopción por Londres del Libro Blanco de 1939, Gran Bretaña no se retiró de Palestina sin antes asegurar el compromiso adquirido en la Declaración Balfour (1917) de implementar el proyecto colonial sionista en dicho territorio (Pappé 1993; Thompson 2019).

En segundo lugar - entiende Ramos Tolosa-, no menos responsable en el fracaso de la mediación de Azcárate fue la opción adoptada por Estados Unidos y la Unión Soviética. Basta con señalar que la Resolución 181 (II) de partición de Palestina contó con el imprescindible apoyo de las dos superpotencias. Ambas, como señalara el profesor Roberto Mesa $(1978,25)$, imponían su voluntad en sus respectivas zonas de influencia, pero en este caso, y por diferentes motivaciones, se pusieron "de acuerdo en un extremo": la creación del Estado israelí. Si en el caso de Estados Unidos era evidente el creciente poder de lo que sería conocido como el lobby proisraelí (Mearsheimer y Walt 2007; Petras 2006), en el de la Unión Soviética se buscaba ganar cierta presencia e influencia en una zona fronteriza (limitaba al sur con Turquía e Irán) en la que estaba vetada por las monarquías tradicionalistas, ultraconservadoras y bajo la fuerte ascendencia de las potencias decimonónicas europeas (Golan 1990, 29).

Pese a que no es el objeto central de su investigación, Ramos Tolosa aporta interesantes pistas históricas y políticas sobre las que es posible seguir indagando respecto al apoyo soviético, mucho menos conocido que el estadounidense. En concreto, muestra cómo la incipiente Guerra Fría no se vertebró de manera clásica en este conflicto, al menos no en un primer momento, en que cada superpotencia apoyaba a los distintos bandos enfrentados; por el contrario, la rivalidad se fraguó en torno al "patrocinio del movimiento sionista" (Ramos 2019, 46), con la esperanza de granjearse un aliado prometedor en una región del alto valor geoestratégico y económico como el Cercano y el Medio Oriente. De hecho, ambas superpotencias reconocieron el Estado israelí de manera casi inmediata en 
mayo de 1948, Washington de facto y, más adelantado, Moscú de iure.

A su condición de superpotencias se sumaba la responsabilidad de ocupar, ambas, dos asientos permanentes en el Consejo de Seguridad de la onU, el máximo órgano responsable de velar por la paz y la seguridad internacional. Por diferentes motivos, se opusieron al despliegue de una fuerza internacional en Palestina como reclamaba, con bastante acierto, Azcárate, para evitar la escalada del anunciado conflicto ante la prevista partición, y propiciar una tregua que permitiera mediar, pero también implementar la resolución de partición. Por el contrario, pese a que ninguna cancillería desconocía el rumbo de los acontecimientos, se dejó a las partes a merced de sus fuerzas (Ramos 2019, 173). La superioridad militar del movimiento sionista se impuso sobre el terreno más allá del territorio otorgado por la ONU (de 56 pasó a 78\%), y acometió un importante desalojo de las poblaciones palestinas (ciudades $\mathrm{y}$ aldeas) en un planificado ejercicio de limpieza étnica, documentada por diferentes historiadores palestinos e israelíes (Morris 1987; Khalidi 1992; Masalha 1992; Pappé 2008; Abdo y Masalha 2018).

En tercer lugar, Ramos Tolosa destaca la responsabilidad de la propia institución internacional, la ONU, al advertir un deliberado descuido de su principal cometido en la preservación de la paz, en particular en dos momentos que considera claves por cuanto podían "haber evitado la escalada bélica y la violación de una tregua” (Ramos, 2019, 428): primero, cuando se aproximaba el final del Mandato británico y no adoptó ninguna medida para implementar la partición, sino que dejó a las partes expuestas a sus asimétricas fuerzas; $y$, segundo, cuando el ejército israelí rompió la tregua durante la primera guerra árabe-israelí en octubre de 1948. Estas aportaciones, consideradas por Ramos Tolosa como las principales de su obra, que indagan el papel crucial de la ONU al enfrentarse a la primera crisis internacional de esa naturaleza, no deben ignorar que el comportamiento de las instituciones internacionales refleja 
el de sus Estados miembros, y también los consiguientes equilibrios de poder en el sistema internacional del que forman parte dichos Estados e instituciones.

Ramos Tolosa también expone la parcialidad de órganos y personalidades de esta institución internacional a la hora de abordar la cuestión de Palestina. Entre otros ejemplos está la mayor comparecencia de la delegación sionista (cuatro veces) en comparación con la palestina (dos veces) ante la Asamblea General de la ONU en 1947 (Ramos 2019, 73); el desequilibrio entre los integrantes del Comité Especial de las Naciones Unidas para Palestina (UNSCOP, por sus siglas en inglés), ya que algunos desconocían la situación o eran abrumadoramente partidarios de la partición incluso antes de viajar a Palestina (78); o bien, la evidente simpatía por el sionismo del propio secretario general de la ONU, el noruego Trygve Lie (1946-1952), y de su asesor militar, el coronel, también noruego, Roscher Lund, que, al parecer, según fuentes secundarias utilizadas por el autor, facilitaron información confidencial a la Agencia Judía (155-156).

Por último, en cuarto lugar, los actores locales y regionales también compartían una importante responsabilidad en el fracaso de la mencionada mediación. Los Estados integrantes de la Liga Árabe, que recientemente se habían independizado, se mostraron débiles, divididos y desconfiados, en particular el rey Abdallah de Transjordania, que acordó con los dirigentes sionistas el reparto de Palestina tras su partición territorial y la expansión de su reinado a expensas de la creación de un Estado árabe-palestino. A su vez, el movimiento sionista -como señala Avi Shlaim- partió de una táctica flexible y de una estrategia firme con la que cosechó importantes triunfos, pero a medida que se fue empoderando, se mostró cada vez más intransigente (Shlaim 1988, 10). Un ejemplo elocuente fue que, tras su proclamación de independencia, reconocimiento internacional e ingreso a la ONU como Estado miembro, Israel se opuso sistemáticamente al retorno de los refugiados palestinos, lo que contravenía la Resolución 194 de la Asamblea General del 11 
de diciembre de 1948. Por su parte, el MNP mostró tanto una táctica como una estrategia inflexibles y careció de algunas dosis de pragmatismo para adaptarse a las cambiantes circunstancias - por ejemplo, para colaborar con la delegación del unsCOP-, además de perderse en rivalidades internas o de anteponer en algunos casos intereses particulares (personalistas o partidistas) sobre los colectivos.

Además de estas conclusiones, cabe extraer otras lecciones tanto del texto del profesor Ramos Tolosa como de las obras referidas y, en general, de la ingente literatura especializada sobre este conflicto (y que por razones de espacio no se pueden referenciar aquí). Con este propósito de avanzar más allá de las conclusiones particulares de cada obra y de sus respectivas aportaciones, se podrá convenir en que hay tres grandes pautas de comportamiento reiteradas a lo largo del tiempo.

Primero, una considerable asimetría de poder entre los dos principales actores enfrentados por el establecimiento de un Estado independiente y soberano en Palestina, a saber, el movimiento nacional palestino y el movimiento sionista, y que se concreta en los diferentes recursos disponibles en capital humano, materiales, económicos, organizativos, movilizadores y comunicativos, unidos al valioso respaldo internacional. Segundo, el apadrinamiento, la parcialidad y la complicidad de la potencia mundial predominante en el sistema internacional de entonces, Gran Bretaña, como potencia colonial y mandataria en Palestina. Sin ese sustancial apoyo hubiera sido prácticamente imposible la materialización de la empresa colonial sionista. Por último, tercero, la endeblez y la maleabilidad de las instituciones internacionales ante el poder y la influencia de las grandes potencias mundiales en función de sus intereses, sus alianzas y sus objetivos, que se advierte tanto en la Sociedad de Naciones como en la entonces incipiente ONU.

¿Cómo se proyectan hasta la actualidad estas tres tendencias, persistentes desde entonces a pesar del tiempo transcurrido? Primera: la asimetría de poder. Ciertamente, la sociedad 
palestina, pese a que fue objeto de una trascendental limpieza étnica y el consiguiente memoricidio, ha logrado recrear y mantener vivas sus señas de identidad nacional ante la reiterada adversidad. Los innegables éxitos políticos y diplomáticos de su movimiento nacional no se han traducido en la creación de un Estado palestino. Actualmente, debilitado y dividido, pasa por sus horas más bajas. Sus recursos de poder son insignificantes e incomparablemente inferiores a los que posee el Estado israelí, considerado por algunas fuentes como la octava potencia en el ranking mundial. En 2017, Israel ingresó en la lista de las principales potencias mundiales en la octava posición, después de Estados Unidos, China, Japón, Rusia, Alemania, India e Irán (Russell y Keeley 2017), y se estima que nunca en su historia ha gozado de un poder y una prosperidad semejantes a los que posee ahora (Tsir y Katz 2019).

Segunda: la protección y la parcialidad de la principal potencia mundial. La especial alianza entre Estados Unidos e Israel ha quedado evidenciada una vez más durante la presidencia de Donald Trump (2017-2021), que ha ido más lejos que otras administraciones al reconocer a Jerusalén como la capital de Israel y la anexión israelí de los Altos del Golán, así como al manifestar su predisposición a un futuro reconocimiento de la anunciada anexión de Cisjordania (anexionada de facto). Así se deriva de su plan de paz, el "acuerdo del siglo", confeccionado según las exigencias y los intereses israelíes, con una evidente exclusión de la parte palestina (cierre de la oficina de la Organización para la Liberación de Palestina en Washington en 2018) y de cualquier referencia al derecho internacional o a las resoluciones de la ONU respecto a la cuestión de Palestina.

Por último, tercera: la endeblez y la maleabilidad de las instituciones internacionales. Sin ninguna contraparte ni contrapeso mundial, las instituciones internacionales muestran claras limitaciones para intentar cambiar este estado de cosas, sin olvidar que son objeto de represalias cuando no se someten al silencio o a esos criterios de subordinación y, por 
el contrario, emprenden iniciativas más resolutivas. Así ha sucedido con algunas agencias especializadas de la ONU, como la Unesco. Después de su reconocimiento de Palestina como Estado miembro (2011), la administración de Obama recortó su aportación económica en 2011, y a finales de 2017 la de Trump anunció su retirada de dicha organización. En esta misma línea, en represalia a la Autoridad Palestina por no aceptar el plan de Trump, a partir de 2018 Washington recortó sustancialmente (en unos 300 millones de dólares) su aportación económica a la Agencia de Naciones Unidas para los Refugiados de Palestina en Oriente Próximo (UNRWA, por sus siglas en inglés), que se ocupa de unos 5.4 millones de refugiados a los que provee servicios y asistencia (educación, sanidad y servicios sociales, principalmente) en la Franja de Gaza, Cisjordania, Jerusalén Este, Líbano, Siria y Jordania.

En conclusión, todo indica que estas pautas se han mantenido con inquebrantable regularidad. De hecho, la ausencia de variaciones significativas en ese comportamiento explica la prolongada irresolución del conflicto. El resultado ha sido la imposición de una política de expansión colonial, de "hechos consumados" sobre el terreno, que reproduce pautas históricas de teoría y comportamiento colonial (Masalha 2000). En este caso, de una colonia de asentamiento o población que, como el nombre lo indica, no estuvo diseñada para explotar los recursos del país ni la mano de obra nativa, como en las colonias de factorías, sino pensada para apropiarse del país y desplazar a la población autóctona por otra foránea. La política colonial y de apartheid israelí puede parecer un anacronismo en el mundo actual, pero no por ello deja de ser menos cruenta y real. Sin un notable apoyo, connivencia, indiferencia o impotencia internacional tampoco se explicaría esta anomalía que se pretende normalizar. Puede parecer un esbozo un tanto determinista, pero si se sustituye el término determinista por poder en las relaciones internacionales, entonces se advertirá la relevancia del argumento. 


\section{Referencias}

Aвdo, Nahla y Nur Masalha, eds. 2018. An Oral History of the Palestinian Nakba. Londres: Zed Books.

AzcÁrate, Pablo de. 1968. Misión en Palestina: nacimiento del Estado de Israel. Madrid: Tecnos.

Golan, Galia. 1990. Soviet Policies in the Middle East: From World War II to Gorbachev. Cambridge: Cambridge University Press.

Khalidi, Rashid. 2007. The Iron Cage. The Story of the Palestinian Struggle for Statehood. Oxford: Oneworld Publications.

Khalid, Walid, ed. 1992. All that Remains: The Palestinian Villages Occupied and Depopulated by Israel in 1948. Washington, D.C.: Institute for Palestine Studies.

Masalha, Nur. 1992. Expulsion of the Palestinians: The Concept of "Transfer" in Zionist Political Thought, 1882-1948. Washington, D.C.: Institute for Palestine Studies.

Masalha, Nur. 2000. Imperial Israel and the Palestinians. The Politics of Expansion. Londres: Pluto Press.

MEARSHEIMER, John J. y Stephen M. Walt. 2007. El lobby israeli y la politica exterior de Estados Unidos. Madrid: Taurus.

Mesa, Roberto. 1978. La lucha de liberación del pueblo palestino. Madrid: Cupsa.

Morris, Benny. 1987. The Birth of the Palestinian Refugee Problem 1947-1949. Cambridge: Cambridge University Press.

PAPpé, Ilan. 1993. Britain and the Arab-Israeli Conflict, 1948-51. Londres: MacMillan. https://doi.org/10.1007/978-1-349-19326-4

Pappé, Ilan. 2008. La limpieza étnica de Palestina. Traducido por Luis Noriega. Barcelona: Crítica.

Petras, James. 2006. The Power of Israel in the United States. Atlanta: Clarity Press.

Ramos Tolosa, Jorge. 2019. Los años clave de Palestina-Israel. Pablo de Azcárate y la ONU (1947-1952). Madrid: Marcial Pons Historia.

Regan, Bernard. 2017. The Balfour Declaration. Empire, the Mandate and Resistance in Palestine. Londres: Verso.

Russell Mead, Walter y Sean Keeley. 2017. "The Eight Great Powers of 2017". The American Interest. 24 de enero de 2017. https:// www.the-american-interest.com/2017/01/24/the-eight-greatpowers-of-2017/ 
Shlaim, Avi. 1988. Collusion Across the Jordan: King Abdullah, the Zionist Movement, and the Partition of Palestine. Oxford: Clarendon Press.

Thompson, Gardner. 2019. Legacy of Empire. Britain, Zionism and the Creation of Israel. Londres: Saqi Books.

Tsir CoHen, Eyal y Eliora Katz. 2019. "Israel's Power Paradox". The National Interest. 12 de septiembre de 2019. https://nationalinterest.org/print/blog/middle-east-watch/israels-powerparadox-80311

José Abu-Tarbush Quevedo es profesor titular de sociología en la Universidad de La Laguna, Tenerife (España). Es autor de diferentes trabajos sobre la cuestión de Palestina y el mundo árabe e islámico, publicados en revistas especializadas y obras colectivas; entre los más recientes destacan los realizados en coautoría con Javier Granados, "La política exterior de Rusia en Oriente Medio: su intervención en Siria” (Revista Electrónica de Estudios Internacionales, núm. 35, 2018, https://doi.org/ 10.17103/reei.35.05); Isaías Barreñada, Ignacio Álvarez-Ossorio y José Antonio Sanahuja, Entre España y Palestina. Revisión crítica de unas relaciones (Bellaterra, 2018), e Isaías Barreñada, "Emergencia, articulación y declive del Movimiento Nacional Palestino durante el periodo de entreguerras" (Miscelánea de Estudios Árabes y Hebraicos, núm. 69, 2020: 61-99, https://doi. org/10.30827/meaharabe.v69i0.1067).

https://orcid.org/0000-0001-9062-1377 josabu@ull.edu.es 